\title{
Virtual-Pinhole PET
}

\author{
Yuan-Chuan Tai ${ }^{1,2}$, Heyu $\mathrm{Wu}^{1}$, Debashish $\mathrm{Pal}^{3}$, and Joseph A. O'Sullivan ${ }^{4}$ \\ ${ }^{1}$ Department of Radiology, Washington University, St. Louis, Missouri; ${ }^{2}$ Alvin J. Siteman Cancer Center, Washington University School \\ of Medicine, St. Louis, Missouri; ${ }^{3}$ Department of Biomedical Engineering, Washington University, St. Louis, Missouri; and ${ }^{4}$ Department \\ of Electrical and System Engineering, Washington University, St. Louis, Missouri
}

We proposed and tested a novel geometry for PET system design analogous to pinhole SPECT called the virtual-pinhole PET (VP-PET) geometry to determine whether it could provide highresolution images. Methods: We analyzed the effects of photon acolinearity and detector sizes on system resolution and extended the empiric formula for reconstructed image resolution of conventional PET proposed earlier to predict the resolutions of VP-PET. To measure the system resolution of VP-PET, we recorded coincidence events as a ${ }^{22} \mathrm{Na}$ point source was stepped across the coincidence line of response between 2 detectors made from identical arrays of $12 \times 12$ lutetium oxyorthosilicate crystals (each measuring $1.51 \times 1.51 \times 10 \mathrm{~mm}^{3}$ ) separated by $565 \mathrm{~mm}$. To measure reconstructed image resolution, we built 4 VP-PET systems using 4 types of detectors (width, $1.51-6.4 \mathrm{~mm}$ ) and imaged 4 point sources of ${ }^{64} \mathrm{Cu}$ (half-life $=12.7$ $\mathrm{h}$ to allow a long acquisition time). Tangential and radial resolutions were measured and averaged for each source and each system. We then imaged a polystyrene plastic phantom representing a 2.5-cm-thick cross-section of isolated breast volume. The phantom was filled with an aqueous solution of ${ }^{64} \mathrm{Cu}(713 \mathrm{kBq} / \mathrm{mL})$ in which the following were imbedded: 4 spheric tumors ranging from 1.8 to $12.6 \mathrm{~mm}$ in inner diameter (ID), 6 micropipettes (0.7or $1.1-\mathrm{mm}$ ID filled with ${ }^{64} \mathrm{Cu}$ at $5 \times, 20 \times$, or $50 \times$ background), and a 10.0-mm outer-diameter cold lesion. Results: The shape and measured full width at half maximum of the line spread functions agree well with the predicted values. Measured reconstructed image resolution $(2.40-3.24 \mathrm{~mm})$ was $\pm 6 \%$ of the predicted value for 3 of the 4 systems. In one case, the difference was $12.6 \%$, possibly due to underestimation of the block effect from the low-resolution detector. In phantom experiments, all spheric tumors were detected. Small line sources were detected if the activity concentration is at least $20 \times$ background. Conclusion: We have developed and characterized a novel geometry for PET. A PET system following the VP-PET geometry provides high-resolution images for objects near the system's high-resolution detectors. This geometry may lead to the development of special-purpose PET systems or resolution-enhancing insert devices for conventional PET scanners.

Key Words: PET; geometry; pinhole; breast imaging

J Nucl Med 2008; 49:471-479

DOI: 10.2967/jnumed.107.043034

Received Apr. 23, 2007; revision accepted Oct. 4, 2007.

For correspondence or reprints contact: Yuan-Chuan Tai, PhD, Washington University School of Medicine, Campus Box 8225, 510 S. Kingshighway Blvd., St. Louis, MO 63110.

E-mail: taiy@wustl.edu

COPYRIGHT @ 2008 by the Society of Nuclear Medicine, Inc.
$\mathbf{P}$ ET has evolved from a research tool for studying neurologic and cardiac functions of humans ( 1 ) to a clinical diagnostic tool for cancer patients (2), particularly since the introduction of PET/CT technology (3). With the introduction of high-resolution animal PET scanners in the mid1990s $(4,5)$, PET became a driving force behind molecular imaging through in vivo imaging of small animals using positron-emitting radionuclide-labeled biomolecules (G).

Resolution of PET is limited by the positron range of the radionuclide, acolinearity of the annihilation $\gamma$-rays, and intrinsic spatial resolution of the detectors. For whole-body PET scanners with large diameters, the blurring of image resolution due to the acolinearity effect is approximately 2$\mathrm{mm}$ full width at half maximum (FWHM) $(7,8)$, whereas for animal PET scanners this effect may be as small as 0.2mm FWHM. When radionuclides of small positron range (such as ${ }^{18} \mathrm{~F}$ and ${ }^{64} \mathrm{Cu}$ ) are used, the image resolution of most animal PET scanners is predominantly limited by the detector's intrinsic spatial resolution (9).

Although there have been significant improvements in the performance of PET scanners through new scintillation material and better detector design (10), fundamental PET scanner geometry remains unchanged-that is, a group of $\gamma$-ray detectors of the same size is arranged into multiple rings or planes to detect annihilation $\gamma$-rays along different angles through coincidence detection. There is maximal sampling density and minimal parallax error at the center of the field of view (FOV) - hence, the best image resolution is there. Several groups have demonstrated that sampling uniformity and the reconstructed image resolution can be improved by wobbling the system or the object being imaged $(11,12)$ or by introducing a small offset between the center of detector pairs and the center of rotation (COR) of the system (13). However, the geometry of these systems does not deviate from the conventional PET geometry significantly.

This work describes a new approach to PET system geometry, the virtual-pinhole PET (VP-PET), derived from the well-known pinhole SPECT geometry. We first describe the theory of operation for VP-PET. We then analyze the intrinsic spatial resolution of the VP-PET system and validate the formula through experiments. We further measure image resolution of $4 \mathrm{VP}-\mathrm{PET}$ systems through experiments. Finally, we imaged a phantom to demonstrate the potential of 
the VP-PET geometry in future development of specialpurpose PET devices.

\section{MATERIALS AND METHODS}

A typical pinhole SPECT system consists of a planar $\gamma$-camera, a pinhole collimator, and a rotation mechanism that allows projection data to be collected from multiple angles. Figure 1A illustrates a simplified pinhole SPECT system without its rotation mechanism. Radioactivity distribution in an object is projected through the pinhole collimation onto the camera surface. Magnification (minification) can be achieved if the object-to-pinhole distance $(b)$ is smaller (larger) than the collimator cone length $(f)$, with the magnification factor $M=f / b$. The image resolution of a pinhole SPECT system can be approximated by the following equations $(14,15)$ :

$$
\begin{gathered}
R_{\text {Colli }} \approx d \cdot\left(\frac{f+b}{f}\right), \\
R_{\text {System }} \approx \sqrt{R_{\text {Colli }}^{2}+\left(\frac{b}{f} \cdot R_{\text {Det }}\right)^{2}},
\end{gathered}
$$

where $R_{\text {Colli }}$ is the collimator resolution, $d$ is the diameter of the pinhole aperture, $R_{D e t}$ is the detector intrinsic spatial resolution, and $R_{\text {System }}$ is the system resolution. When the object-to-pinhole distance $(b)$ is significantly smaller than the collimator cone length (f), the collimator resolution approaches the size of the pinhole aperture, whereas the system resolution becomes less dependent on the detector intrinsic spatial resolution. The use of pinhole collimator(s) permits high-resolution animal SPECT using $\gamma$-cameras of low intrinsic spatial resolution (16).

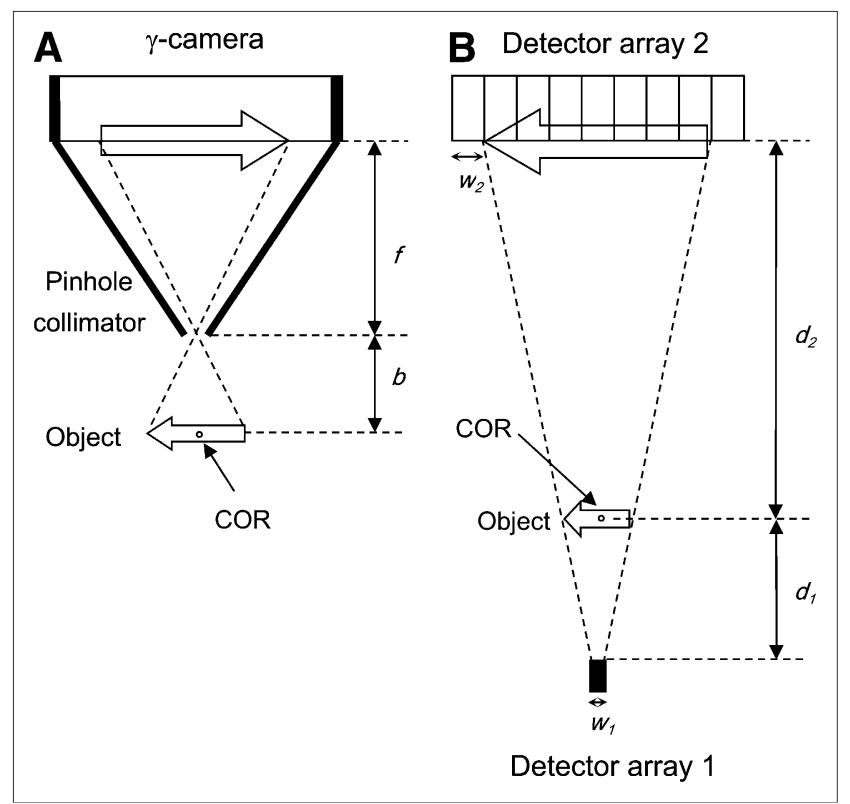

FIGURE 1. (A) Simplified pinhole SPECT system consists of a $\gamma$-camera and a pinhole collimator that can be rotated around a COR. (B) Simplified VP-PET system consists of a high-resolution detector array (1) and a low-resolution detector array (2) that can be rotated around a COR.
A PET system contains one or more pairs of detectors for coincidence detection. Coincidence detection circuits replace the physical collimator to perform electronic collimation. Although most PET scanners were designed to use multiple detectors of the same dimension in a system, one can also use 2 completely different $\gamma$-ray detectors for coincidence detection. Figure 1B illustrates a system with 2 detector arrays of different sizes. Detector array 1 consists of a single detector element of width $w_{l}$. Detector array 2 consists of multiple detector elements of width $w_{2}$. The coincidence lines of response between the detector in array 1 and all detector elements in array 2 form a fanbeam geometry. If the detector pair in Figure $1 \mathrm{~B}$ is rotated around the object located at the COR, coincidence events can be collected from multiple angles and reconstructed using fanbeam reconstruction algorithms to obtain tomographic images.

If one considers the detector array 1 in Figure $1 \mathrm{~B}$ as an $\mathrm{x}$-ray source and the array 2 as part of a full ring of $x$-ray detectors, this system approximates the geometry of a fourth-generation (4G) CT scanner (17). The fundamental difference between a VP-PET system and a CT system is that the signal carrier in PET is the annihilation $\gamma$-rays originating from the object, whereas for CT the signal carrier is the $\mathrm{x}$-ray flux originating from the $\mathrm{x}$-ray tube. If one considers the fanbeam geometry in Figure 1B, the closer the object is to the detector array 1, the larger the object is projected onto the surface of detector array 2 . The magnification factor based solely on geometry is $M=\left(d_{l}+d_{2}\right) / d_{l}$, which is the same as for a CT system. However, unlike a CT system where the focal spot of an X-ray tube is typically considered to be a point source, the detector in array 1 has a finite width, $w_{l}$. Therefore, the image resolution of such a PET system is a function of detector widths $\left(w_{1}\right.$ and $\left.w_{2}\right)$ and object-todetector distances $\left(d_{l}\right.$ and $\left.d_{2}\right)$. This is similar to a pinhole SPECT system whose image resolution depends on several system parameters as described in Equations 1 and 2. Hence, we have named the geometry in Figure 1B the "Virtual-Pinhole" PET geometry and systems that follow this geometry VP-PET systems. We characterize the resolution of a VP-PET system through analyses of the intrinsic spatial resolution between 2 detectors of different dimensions, followed by measurement of image resolution of VP-PET systems of 4 different configurations.

\section{Resolution for VP-PET Geometry}

Among the 3 factors (positron range, acolinearity, and detector intrinsic spatial resolution) that limit the spatial resolution of an annihilation coincidence detection system (or a PET system), the latter 2 can depend on the system design and, therefore, so were analyzed for a VP-PET system.

Theoretic Derivation. The effect on spatial resolution due to acolinearity of the annihilation $\gamma$-rays is commonly expressed as $\mathrm{R}_{180^{\circ}}=0.0022 \cdot D$, where $D$ is the diameter of a PET scanner or the distance between the detectors. This formula represents the FWHM of the blurring profile at the center between 2 detectors due to angular distribution of the annihilation photon acolinearity, which is approximately $0.5^{\circ}(0.0088 \mathrm{rad})$ FWHM (18). For the geometry in Figure 1B, the acolinearity effect on spatial resolution can be expressed as (in FWHM):

$$
R_{180^{\circ}}=0.0088 \cdot \frac{d_{1} \cdot d_{2}}{\left(d_{1}+d_{2}\right)}
$$

This term can be significantly smaller than the commonly accepted expression, $\mathrm{R}_{180^{\circ}}=0.0022 \cdot D$, when $d_{l}$ is significantly 
smaller than $d_{2}$ - that is, when the object is very close to one of the detectors.

Intrinsic spatial resolution of a pair of detectors of the same dimension (Fig. 2A) has been thoroughly explored $(15,19)$. The detection profile is symmetric around the center between the 2 detectors with its shape approaching a triangle at the center and a rectangle near both ends. For locations other than these 2 positions, the resolution profile approaches a trapezoid function. The
Derenzo et al. (20) needs to be included in the quadratic sum in Equation 7 to describe the blurring effect due to the use of block detectors in a VP-PET system. Combining the system resolution in Equation 7 and the empiric formula proposed by Moses and Derenzo that describes the reconstructed image resolution of a conventional PET scanner using cylindric geometry, we obtain the following expression for the reconstructed image resolution of a VP-PET system near its COR:

$$
R_{\text {img }} \approx 1.25 \cdot \sqrt{R_{s r c}^{2}+\left[0.0088 \cdot \frac{d_{1} \cdot d_{2}}{\left(d_{1}+d_{2}\right)}\right]^{2}+\left[\frac{d_{2} \cdot w_{1}+d_{1} \cdot w_{2}+\left|d_{2} \cdot w_{1}-d_{1} \cdot w_{2}\right|}{2 \cdot\left(d_{1}+d_{2}\right)}\right]^{2}+B E^{2}}
$$

width of the base of the detection profiles is defined by the width of the detectors, $w$, whereas the width of the plateau is defined by the 2 dashed lines connecting the 2 detectors - that is, the width of the plateau changes from zero near the center of the system to $w$ near the edge of the system.

For a coincidence detection system that uses detectors of 2 different sizes (Fig. 2B), the detection profile is asymmetric. The width of the base of the profiles is still defined by the detector widths, but is no longer constant, instead approaching $w_{1}$ and $w_{2}$ at the 2 ends. The width of the plateau is still defined by the 2 dashed lines connecting the 2 detectors, but the location where plateau width equals zero is not at the center and is instead near the smaller detector. Given separation $D$ between 2 detectors, it can be shown that the width of the base, $w_{\mathrm{b}}$, the width of the plateau, $w_{\mathrm{p}}$, and the FWHM of the detection profile, $R_{d e t}$, at distance $x$ from detector 1 are:

$$
\begin{gathered}
w_{\mathrm{b}}=w_{1}+\frac{w_{2}-w_{1}}{D} \cdot x=\frac{(D-x) \cdot w_{1}+x \cdot w_{2}}{D} . \\
w_{\mathrm{p}}=\frac{\left|(D-x) \cdot w_{1}-x \cdot w_{2}\right|}{D} . \\
R_{\mathrm{det}}=\frac{w_{b}+w_{p}}{2}=\frac{(D-x) \cdot w_{1}+x \cdot w_{2}+\left|(D-x) \cdot w_{1}-x \cdot w_{2}\right|}{2 \cdot D} .
\end{gathered}
$$

Eq. 6

For a VP-PET system following the geometry in Figure 1B with its COR located at $d_{1}$ from detector array 1 and $d_{2}$ from detector array 2, the system resolution near the center of the FOV can be expressed as: where $B E$ is the block effect and the factor 1.25 accounts for the difference between system resolution and reconstructed image resolution due to insufficient sampling and the use of the filteredbackprojection algorithm.

Intrinsic Spatial Resolution Measurements. To investigate the intrinsic spatial resolution of a VP-PET system, we set up a coincidence detection system using 2 identical PET detectors separated by $565 \mathrm{~mm}$. This distance represents a potential VPPET application when high-resolution detectors are positioned at $159 \mathrm{~mm}$ from the center of the FOV inside a clinical whole-body PET scanner of 406-mm radius. Each detector contains a lutetium oxyorthosilicate (LSO) array directly coupled to a position-sensitive photomultiplier tube ([PS-PMT] R5900-C12; Hamamatsu Photonics, Japan). The LSO array has $12 \times 12$ crystals each measuring $1.51 \times 1.51 \times 10 \mathrm{~mm}^{3}$ with a pitch of $1.6 \mathrm{~mm}$ in both directions. The position of an event was determined by a ratiometric positioning algorithm commonly used in microPET detectors $(21,22)$. A sealed ${ }^{22} \mathrm{Na}$ point source was positioned at various distances between the 2 detectors and stepped across the coincidence lines of response with a step size of $0.177 \mathrm{~mm}$. Coincidence events were recorded at each source location. In postprocessing, coincidence events between a single crystal of the left detector module and a group of 4-by-4 crystals of the right detector module were counted and plotted as a function of the point source location. This effectively measured the line spread function of a pair of annihilation coincidence detection detectors of different dimensions $\left(1.51 \times 1.51 \times 10 \mathrm{~mm}^{3}\right.$ vs. $\left.6.31 \times 6.31 \times 10 \mathrm{~mm}^{3}\right)$ at different locations along the coincidence line of response. The FWHM and full width at tenth maximum (FWTM) were calculated from each line spread function by linear interpolation between 2 nearest measured values bracketing the half-values and one-tenth values of the peak, respectively.

$$
R_{s y s} \approx \sqrt{R_{s r c}^{2}+R_{180^{\circ}}^{2}+R_{\mathrm{det}}^{2}} \approx \sqrt{R_{s r c}^{2}+\left[0.0088 \cdot \frac{d_{1} \cdot d_{2}}{\left(d_{1}+d_{2}\right)}\right]^{2}+\left[\frac{d_{2} \cdot w_{1}+d_{1} \cdot w_{2}+\left|d_{2} \cdot w_{1}-d_{1} \cdot w_{2}\right|}{2 \cdot\left(d_{1}+d_{2}\right)}\right]^{2}}
$$

where $R_{s r c}$ is the effective source dimension that includes the positron range effect. Because Equation 7 is derived from 2 individual detectors in Figure 2B, an additional term similar to the "block effect" that was suggested by Moses and Derenzo (7) and
The FWHM was plotted against the source-to-detector distance and compared with the resolutions predicted by Equation 7. In the calculation, the effective source dimension was assumed to be $0.8-\mathrm{mm}$ FWHM based on its nominal diameter of $0.5 \mathrm{~mm}$ and the 


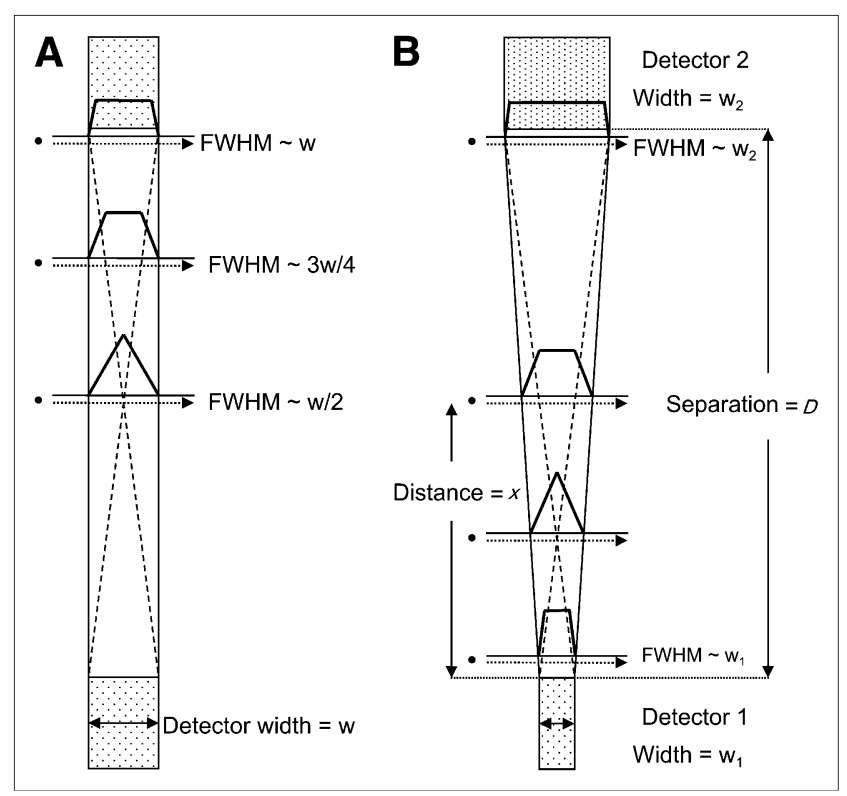

FIGURE 2. Intrinsic spatial resolution of a pair of PET detectors in coincidence (shown as thick solid lines in drawing) is a function of source location and detector width(s). (A) For conventional PET system using 2 identical detectors, the profile approaches a triangle and a trapezoid at center and edge of system, respectively. (B) For VP-PET system, widths of the base (or plateau) of resolution profiles are defined by solid lines (or dashed lines) connecting the 2 detectors. FWHM of the profiles can be approximated by one half of the sum of widths of the plateau and the base.

relatively small positron range for ${ }^{22} \mathrm{Na}$. Because the dimension and the charge-division readout of our detectors are similar to those used in a commercial animal PET scanner (microPET R4; Siemens Medical Solutions, Inc.), we expect the block effect of these detectors to be similar to that of the microPET scanner. Tomic et al. (23) recently showed that the block effect is approximately 1.7-mm FWHM for a clinical PET scanner (ECAT HR+; Siemens Medical Solutions, Inc.) and 0.8-mm FWHM for the microPET R4 system. Therefore, we assumed a block effect of $0.8-\mathrm{mm}$ FWHM for our detectors and calculated the predicted resolution with and without the inclusion of block effect in Equation 7.

Reconstructed Image Resolution Measurements. We evaluated the image resolution of VP-PET systems via the experimental setup in Figure 3A to mimic the VP-PET geometry in Figure 1B. Detectors of different sizes were mounted on 2 concentric rotation stages that can be rotated independently for a full $360^{\circ}$ to acquire coincidence events from all angles. On the basis of a 2-dimensional coordinate system with its origin located at the COR, a high-resolution detector (array 1) was positioned with its front surface centered at $(-147,0)$ (in $\mathrm{mm}$ ), whereas a low-resolution detector (array 2) was positioned with its front surface centered at $(0,400)$. Detector array 1 (left) was either an array of $8 \times 8$ LSO crystals each measuring $2.2 \times 2.2 \times 10 \mathrm{~mm}^{3}$ or an array of $12 \times 12$ LSO crystals each measuring $1.51 \times 1.51 \times 10 \mathrm{~mm}^{3}$. Scintillation light was detected by the same type of PS-PMT as already described. These types of detectors are commonly used in highresolution animal PET systems $(22,24)$. Detector array 2 (right) was either an array of $8 \times 8$ LSO crystals each measuring $6.4 \times$ $6.4 \times 25 \mathrm{~mm}^{3}$ or an array of $13 \times 13$ LSO crystals each measuring
$4.0 \times 4.0 \times 20 \mathrm{~mm}^{3}$. Scintillation light was detected by 4 units of single-channel PMT. These types of detectors are commonly used in clinical PET/CT scanners.

We constructed 4 point sources using micropipettes of $0.5-\mathrm{mm}$ inner diameter (ID) and 1.0-mm outer diameter (OD). Each source contained approximately $18.5 \mathrm{MBq}$ of ${ }^{64} \mathrm{Cu}$ solution. ${ }^{64} \mathrm{Cu}$ has a half-life of $12.7 \mathrm{~h}$, permitting data to be collected from multiple angles over an extended time. The presence of prompt $\gamma$-rays from ${ }^{64} \mathrm{Cu}$, however, could be in coincidence with the annihilation $\gamma$-rays and result in effects that are similar to scatter coincidences that could degrade the FWTM of the resolution measurement slightly. The sources were positioned at $(-9,0),(11,0),(1,4)$, and $(1,-6)$ (in $\mathrm{mm})$. In each of the 4 configurations, the sources were imaged through step-and-acquire motion (Fig. 3B). Detector array 1 was rotated to 48 positions to cover $360^{\circ}$ and provide full $2 \pi$ sinograms. Each time detector array 1 was moved, detector array 2 was moved to 3 positions to cover all 4 point sources. Coincidence events collected at different angles were sorted to form fanbeam sinograms assuming the high- and low-resolution crystals were uniformly distributed along the 2 circles in Figure 3B without considering the depth of interaction.

To reconstruct the images, we implemented a filtered-backprojection algorithm based on the work by Besson (25) that was originally developed for the 4G CT geometry. In contrast to the original algorithm that assumes the $\mathrm{x}$-ray source to be an infinitely small point source, the refined algorithm incorporates the width of the detectors in both arrays 1 and 2 in the calculation of the system matrix to better represent the geometry of VP-PET systems (26). Data were not normalized for detector efficiency nor were corrected for attenuation. The reconstruction filter was a ramp filter cutoff at the Nyquist frequency. From the reconstructed images, tangential and radial resolution were measured for the 4 line sources and then averaged to obtain the average image resolution of VP-PET systems near their centers of FOV.

Image resolution of the 4 VP-PET systems was also calculated using Equation 8. It was assumed that detectors 1 and 2 are separated by a fixed distance of $547 \mathrm{~mm}$. The COR location was varied from the surface of detector 1 to the surface of detector 2 . The effective source dimension was assumed to be $0.8-\mathrm{mm}$ FWHM for the ${ }^{64} \mathrm{Cu}$ point sources. The block effect was also assumed to be $0.8-\mathrm{mm}$ FWHM. The calculated image resolution of the 4 VP-PET systems was plotted as a function of COR location between the 2 detectors.

\section{Phantom Study}

A piece of polystyrene plastic $\left(20 \times 18 \times 2.5 \mathrm{~cm}^{3}\right)$ was carved to form a cavity that has a shape that resembles the cross-section of an isolated breast volume. The cavity was filled with $170 \mathrm{~mL}$ of aqueous solution of ${ }^{64} \mathrm{Cu}(713 \mathrm{kBq} / \mathrm{mL})$ to mimic background activity in tissues. This unusually high-activity concentration was used because the experiment requires 720 detector positions to completely cover the phantom using only 2 detector arrays. The acquisition time at each detector position was very short and, thus, requires high-activity concentration to obtain a reasonable number of counts. Four fillable spheres of different IDs (12.6, 10.0, 5.6, and $1.8 \mathrm{~mm}$ ) were filled with an aqueous solution of ${ }^{64} \mathrm{Cu}$ at an activity concentration of $3.56 \mathrm{MBq} / \mathrm{mL}$ (5× background) and inserted into the cavity to mimic tumors with high uptake. Another fillable sphere of 10.0-mm OD was filled with water to mimic a cold lesion in warm background. Six micropipettes (3 of 1.1-mm ID and 2.0-mm OD, and 3 of $0.7-\mathrm{mm}$ ID and 1.6-mm OD) were filled with an aqueous solution of ${ }^{64} \mathrm{Cu}$ at 3 different activity concentrations $(5 \times, 20 \times$, and 


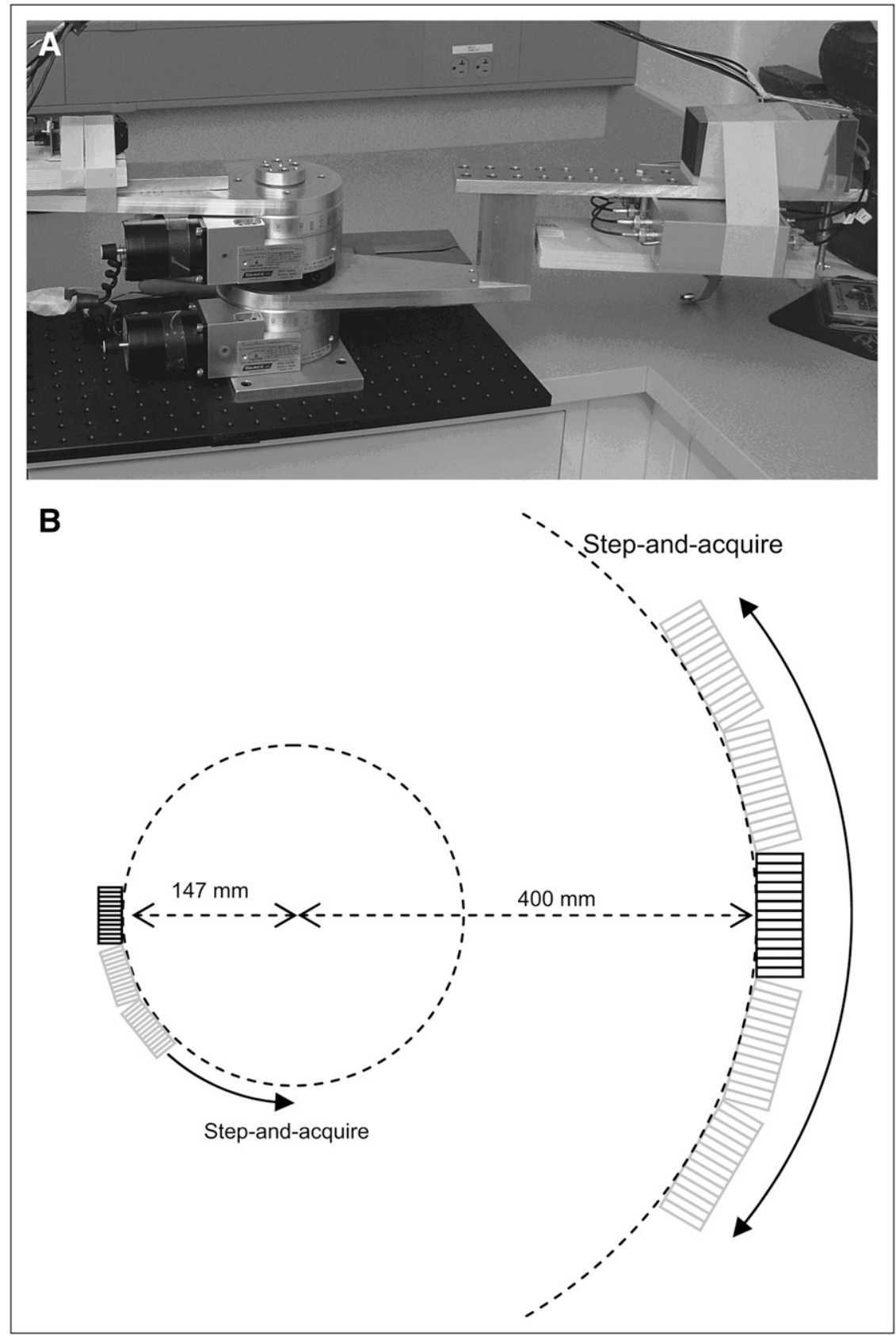

FIGURE 3. (A) VP-PET system was set up using high-resolution detectors (array 1) on left and low-resolution detectors (array 2) on right. (B) A typical scan requires multiple step-and-acquire motions. In this particular setup, detector-to-center distances $\left(d_{1}\right.$ and $d_{2}$ of Fig. $\left.1 \mathrm{~A}\right)$ were 147 and $400 \mathrm{~mm}$, respectively.
$50 \times$ background) to mimic 65 -mm-long line sources. The locations of all tumor inserts and micropipettes in the phantom are shown in Figure 4.

The entire phantom was centered in a VP-PET system similar to Figure $3 \mathrm{~A}$. The detector array 1 was based on LSO crystals of $1.51 \times$ $1.51 \times 10 \mathrm{~mm}^{3}$. The detector array 2 was based on LSO crystals of $4.0 \times 4.0 \times 20 \mathrm{~mm}^{3}$. The distances from the COR to the 2 detectors remained 147 and $400 \mathrm{~mm}$, respectively. Coincidence events were collected after the step-and-acquire motion. Detector array 1 was rotated to 48 positions to cover $360^{\circ}$. Each time detector array 1 rotated to a new position, detector array 2 was rotated to 15 positions to cover the entire phantom. It took $15 \mathrm{~h}$ to collect data from 720 positions, with the acquisition time at each location adjusted for radioactive decay. Coincidence events collected at different locations were sorted to form fanbeam sinograms. The same filtered-backprojection algorithm described previously was used to reconstruct 20 slices of tomographic images across the phantom. Data were not normalized for detector efficiency nor were attenuation corrected. The reconstruction filter was a ramp filter cutoff at the Nyquist frequency.

\section{RESULTS}

\section{Intrinsic Spatial Resolution of VP-PET Geometry}

Figure 5A shows the line spread functions measured at different locations between 2 detectors of different dimensions. The shapes agree well with those illustrated in Figure 2B. The profiles are no longer symmetric relative to the 


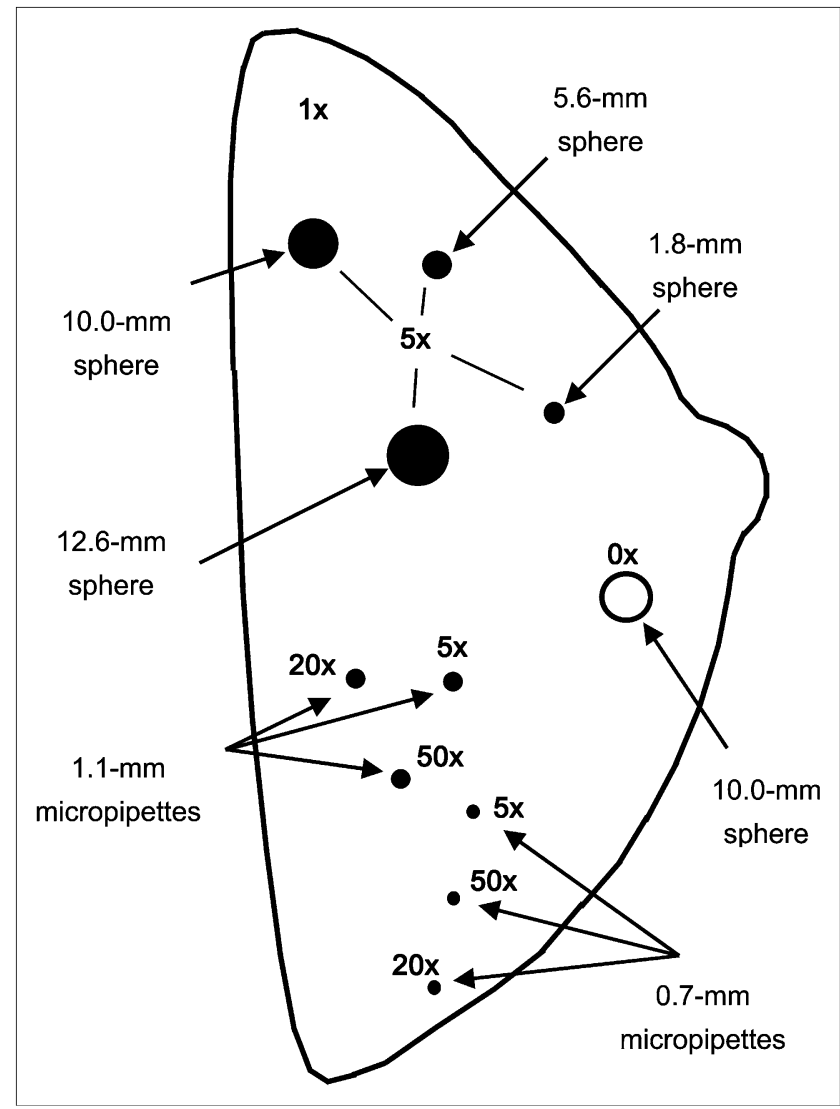

FIGURE 4. Polystyrene plastic breast phantom with fillable background and tumor inserts. Four spheric inserts were filled with ${ }^{64} \mathrm{Cu}$ solution at $5 \times$ background activity concentration. Another spheric insert (10.0-mm OD) was filled with water to mimic a cold lesion. Six micropipettes ( 3 of 1.1-mm ID and 3 of $0.7-\mathrm{mm}$ ID) were filled with $5 \times, 20 \times$, and $50 \times$ background activity concentration. Phantom was imaged by a VP-PET system similar to setup in Figure $3 \mathrm{~A}$.

center between the 2 detector arrays. The FWHM of the profile approaches detector width $w_{1}$ (or $w_{2}$ ) when the point source was near detector array 1 (or 2).

Figure 5B shows good agreement between the FWHM measured from these line spread functions and those predicted by Equation 7 using various source-to-detector distances $\left(d_{1}\right.$ and $\left.d_{2}\right)$. When the block effect is included in Equation 7, there is little change for the right half of the plot (Fig. 5B) in which system resolution is dominated by the intrinsic resolution of array 2. However, near detector array 1 , where the system resolution is roughly 2-mm FWHM, inclusion of the block effect better predicts measured system resolution.

\section{Reconstructed Image Resolution}

Figure 6A shows images of four ${ }^{64} \mathrm{Cu}$ point sources obtained from the 4 VP-PET systems. The measured image resolution of the 4 VP-PET configurations ranged from 2.40- to 3.24-mm FWHM (Table 1).

Figure 6B shows the image resolution (FWHM) calculated by Equation 8 for 4 VP-PET systems as a function of
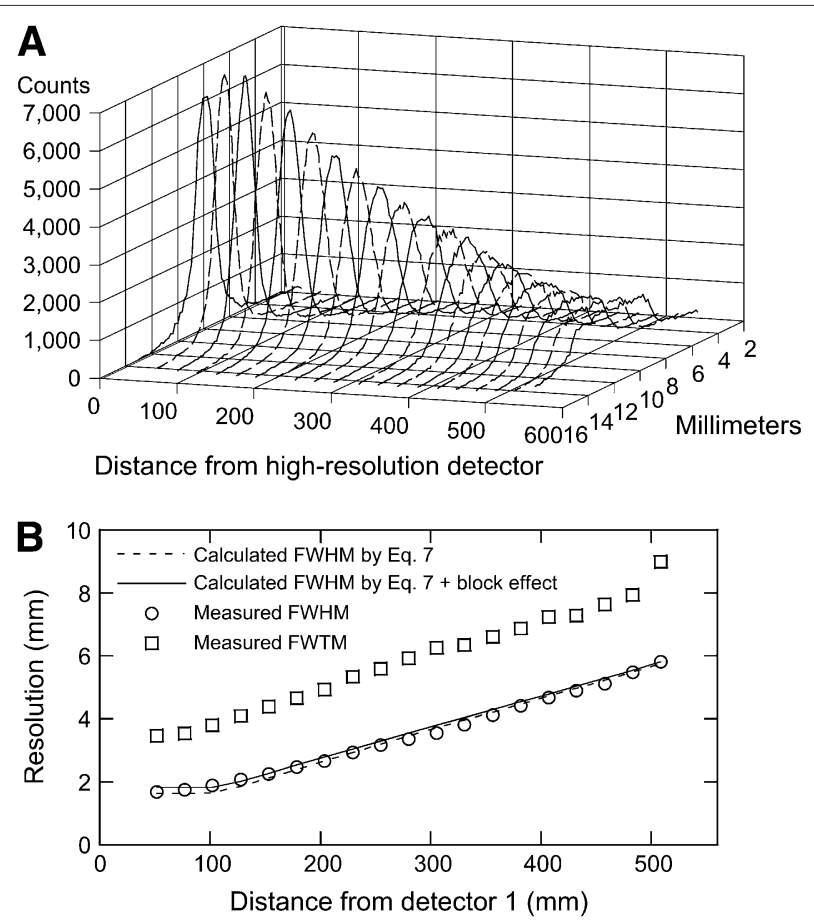

FIGURE 5. (A) Line spread function measured at different locations between 2 detectors resembling intrinsic resolution profiles of VP-PET system. Detectors on left were $1.51 \times 1.51 \times$ $10 \mathrm{~mm}^{3}$ LSO crystals. Detectors on right were $6.31 \times 6.31 \times 10$ $\mathrm{mm}^{3}$ LSO crystals. Separation between 2 detector arrays was $565 \mathrm{~mm}$. (B) FWHM and FWTM measured from profiles in A are plotted as a function of source locations. System resolution (FWHM) of corresponding VP-PET calculated by Equation 7 was included for comparison.

the COR location. The block effect of the detector was assumed to be 0.8-mm FWHM and included in the calculation. The solid vertical line denotes the COR that corresponds to our 4 imaging experiments (i.e., $d_{1}=147 \mathrm{~mm}, d_{2}=400 \mathrm{~mm}$ ). The predicted image resolution of the 4 VP-PET systems ranges from 2.30- to 2.83-mm FWHM (Table 1).

Equation 8 predicted within $6 \%$ the image resolution for 3 of the 4 configurations (cases a, b, and c in Table 1). For case $\mathrm{d}$, the predicted image resolution deviated from the measured value by $12.6 \%$ ( 2.83 vs. $3.24 \mathrm{~mm}$ ). In this configuration, both $w_{1}$ and $w_{2}$ are at their maximum (2.2 vs. $6.4 \mathrm{~mm}$, respectively) compared with the other configurations. Therefore, the 0.8-mm FWHM block effect used in the image resolution calculation was likely to be an underestimation of the actual block effect and may have caused this discrepancy.

\section{Phantom Study}

Figure 7 shows 2 image planes through the phantom. Although these 2 images exhibit artifacts due to the lack of attenuation correction, the high-resolution imaging capability of the VP-PET system detected many of the small lesions. All spheric sources, including the 10.0-mm OD cold spheric lesion and the 1.8-mm ID hot lesion are visible. The 1.1- and 


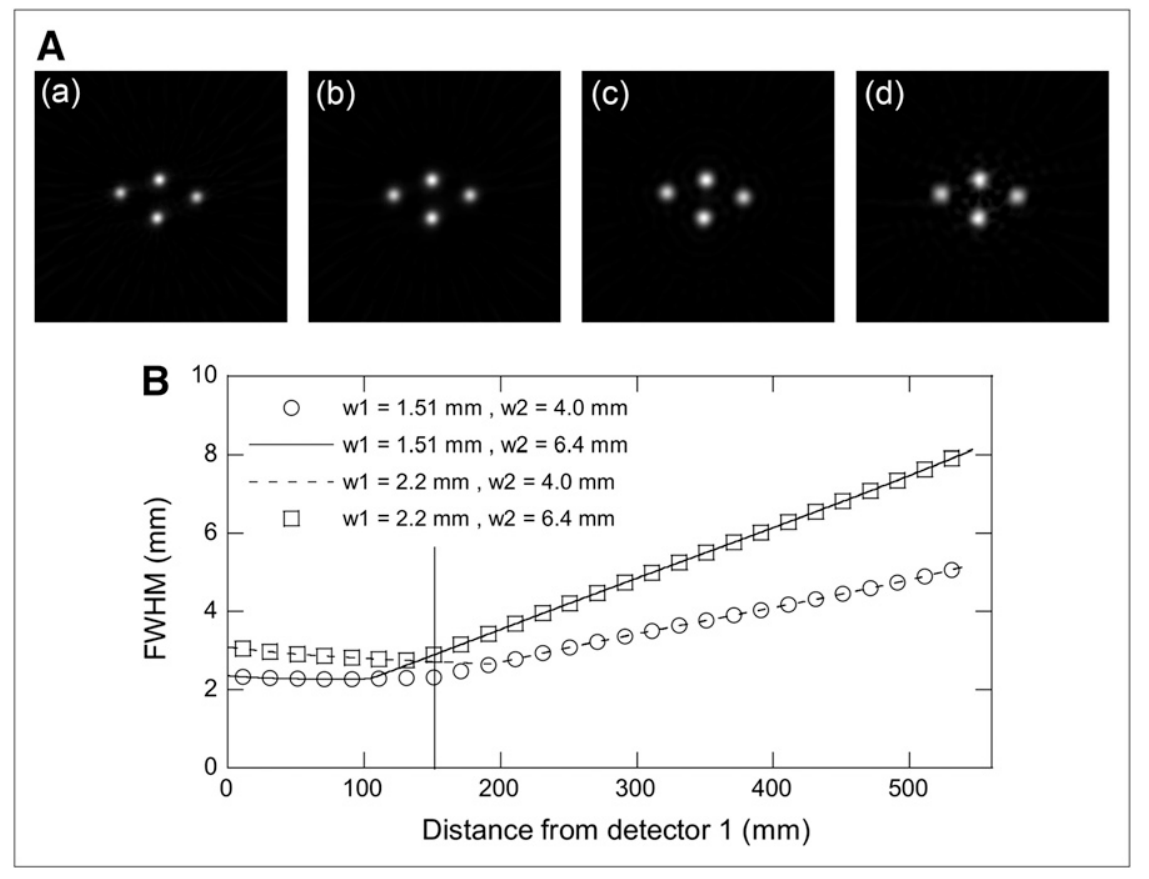

FIGURE 6. (A) Four ${ }^{64} \mathrm{Cu}$ point sources were imaged by 4 VP-PET systems as specified in Table 1. Separation between 2 point sources along $x$-axis was $20 \mathrm{~mm}$. FWHM of 4 point sources was measured in both tangential and radial directions and averaged to obtain the average image resolution for each VP-PET system. (B) Image resolution of 4 VP-PET systems was calculated using Equation 8 and plotted against distance between COR of system and detector 1. Vertical solid line corresponds to setup in which $\mathrm{d}_{1}=147 \mathrm{~mm}$ and $\mathrm{d}_{2}=400 \mathrm{~mm}$.

0.7-mm ID line sources are visible when the tumor-tobackground ratio is 20 or higher.

\section{DISCUSSION}

The derivation of Equations 7 and 8 was based on a VPPET system that uses detectors of 2 different widths, with their front surfaces directly facing each other. It did not consider detectors facing each other at an oblique angle, nor did it factor in the effective depth of $\gamma$-ray interactions that depends on the crystal lengths. Furthermore, the derivation did not consider the type of detector material whose photofraction may impact the positioning accuracy in a detector. Therefore, it is not suggested that Equations 7 and 8 can precisely predict the spatial resolution of a VP-PET system. Rather, these equations represent a logical extension of the empiric formula by Moses and Derenzo (7) that estimates the image resolution near the center of FOV of a conventional PET system. With Equation 8, one may estimate the image resolution of a VP-PET system near its center of the FOV (also its COR) located distance $d_{1}$ from detector 1 and distance $d_{2}$ from detector 2. This should not be confused with the image resolution of a VP-PET system at an arbitrary location within its FOV because the latter largely depends on the crystal length and the associated parallax error, both of which were not modeled here.

It is difficult to predict the block effect theoretically because it would require accurate modeling of all physical processes involved in the $\gamma$-ray detection and electronic processing. Tomic et al. (23) have demonstrated that the block effect can be estimated through a series of measurements and data fitting. However, this approach was developed for conventional PET geometry and is only applicable to sources equidistant between a pair of identical detectors. Because a VP-PET system uses detector blocks of different crystal dimensions, fully characterizing the block effect would require a few thousand measurements at different locations between 2 detector blocks and using detector blocks of different sizes, and doing so is beyond the scope of this work. It is possible that the block effect of individual detectors can be measured conventionally and incorporated into the last term of Equation 7 through the use of "effective crystal width" that combines the physical crystal width with the block effect. However, this can be validated only after extensive measurements and will be left for future investigations. In the Intrinsic Spatial Resolution Measurements section, we used 2 identical detector blocks as array 1 and array 2 and, subsequently, grouped multiple crystals in detector 2 to mimic crystals of larger dimension. We expected the 2 detector blocks to have similar block effects, which later led to good agreement between the predicted resolution and the measured resolution in Figure 5B. In the Reconstructed Image Resolution Measurements section, we assumed that the block effect of detector 1 (0.8-mm FWHM) is the dominant factor because the COR of the 4 VP-PET systems was relatively close to detector 1 . This is likely to be an underestimation of the actual block effect, particularly for systems that use 6.4-mm-wide crystals in detector block 2 .

Figure 5B shows that the intrinsic spatial resolution of a VP-PET system is dominated by $w_{1}$ when the source is near detector 1 and dominated by $w_{2}$ when the source is near detector 2 . The transition point is at a location where $d_{1} / d_{2}=$ $w_{1} / w_{2}$. This can be explained by the term related to detector widths in Equation 7 . When $d_{1} / d_{2}<w_{1} / w_{2}$, the system resolution depends primarily on $w_{1}$. When $d_{1} / d_{2}>w_{1} / w_{2}$, the system resolution depends primarily on $w_{2}$. If $w_{1}=w_{2}$, then 


\begin{tabular}{|c|c|c|c|}
\hline & & \multicolumn{2}{|c|}{ Detector 1} \\
\hline & & $\begin{array}{c}12 \times 12 \text { LSO crystals } \\
1.51 \times 1.51 \times 10.0 \mathrm{~mm}^{3}\end{array}$ & $\begin{array}{c}8 \times 8 \text { LSO crystals } \\
2.2 \times 2.2 \times 10.0 \mathrm{~mm}^{3}\end{array}$ \\
\hline & $\begin{array}{l}13 \times 13 \text { LSO crystals } \\
4.0 \times 4.0 \times 20.0 \mathrm{~mm}^{3}\end{array}$ & $\begin{array}{c}\text { (a) } 2.40 \pm 0.06 \mathrm{~mm} \\
(2.30 \mathrm{~mm})\end{array}$ & $\begin{array}{c}\text { (b) } 2.76 \pm 0.04 \mathrm{~mm} \\
(2.72 \mathrm{~mm})\end{array}$ \\
\hline Detector 2 & $\begin{array}{c}8 \times 8 \text { LSO crystals } \\
6.4 \times 6.4 \times 25.0 \mathrm{~mm}^{3}\end{array}$ & $\begin{array}{c}\text { (c) } 3.00 \pm 0.02 \mathrm{~mm} \\
(2.83 \mathrm{~mm})\end{array}$ & $\begin{array}{c}\text { (d) } 3.24 \pm 0.12 \mathrm{~mm} \\
(2.83 \mathrm{~mm})\end{array}$ \\
\hline
\end{tabular}

Average resolution (FWHM) was measured from 4 point sources imaged by different combinations of detectors. Predicted image resolution of 4 systems was calculated by Equation 8 and is shown in parentheses.

the transition point is at the location where $d_{1}=d_{2}$, which corresponds to the center of a conventional PET system where the intrinsic spatial resolution is at its minimum. In a VP-PET system, the object should be placed near detector 1 to obtain the highest spatial resolution. To sample the object from all angles with high resolution, the COR should also be located near detector 1 rather than at the center between detectors 1 and 2. This is similar to pinhole SPECT where an object is placed near the pinhole collimator and the system is rotated around the object with a fixed object-to-pinhole distance to maintain the same magnification from all angles.

The background activity concentration in the phantom study shown in Figure 7 was $713 \mathrm{kBq} / \mathrm{mL}$. Because the branching ratio for $\beta^{+}$decay is $18 \%$ and $97 \%$ for ${ }^{64} \mathrm{Cu}$ and ${ }^{18} \mathrm{~F}$, respectively, the effective activity concentration in the phantom was $132 \mathrm{kBq} / \mathrm{mL}$ if the radionuclide used was ${ }^{18} \mathrm{~F}$. This activity concentration is approximately 32-fold higher than the activity concentration of ${ }^{18} \mathrm{~F}-\mathrm{FDG}$ in a typical
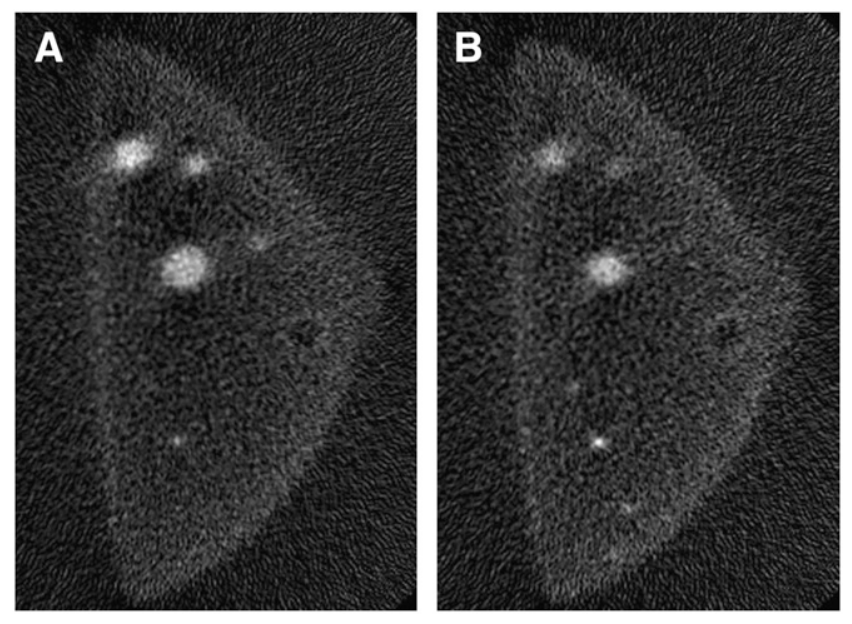

FIGURE 7. Two image planes through a phantom that mimics an isolated breast volume. (A) Image plane that crosses all spheric sources. Four hot spheres (tumor-to-background ratio $=$ 5) and cold spheric lesion are all clearly visible. Smallest spheric source has ID of $1.7 \mathrm{~mm}$. (B) Image plane that crosses the 6 micropipettes. The 1.1- and 0.7-mm (ID) line sources are visible when tumor-to-background ratio is 20 or above. whole-body PET protocol, assuming $370 \mathrm{MBq}$ of ${ }^{18} \mathrm{~F}-\mathrm{FDG}$ are injected and evenly distributed in a 70-kg subject and a 40-min uptake period. In our experiment, the acquisition time at the initial angle was approximately $45 \mathrm{~s}$, which would correspond to a 24-min acquisition time for the first angle with an activity concentration of a typical patient study. However, if one builds a VP-PET system that has large solid angle coverage such that it requires little or no rotation to sample the entire object (27), it is possible to obtain the same level of signal-to-noise ratio and image resolution as was shown in Figure 7 within a reasonable amount of acquisition time.

The potential of VP-PET geometry lies in the fact that one may construct a PET system with different types of detectors and configure the system such that the image resolution is minimally dependent on the low-resolution detectors. There are 2 main disadvantages to such a system. First, the system sensitivity is low compared with a typical PET scanner if only 2 detector blocks are used. Similar to a SPECT system, where one can use a multipinhole collimator to raise the overall system sensitivity, increasing the number of detectors in array 1 will improve the overall system sensitivity of VP-PET systems. Because collimation is performed electronically, the sensitivity of VP-PET is theoretically much higher than that of multipinhole SPECT.

The second drawback is that the system's high resolution is limited to a small imaging FOV. This is, again, very similar to a pinhole SPECT system, where a large magnification and a small usable FOV are used to gain high-resolution imaging capability. For certain imaging applications, such as brain or breast imaging, it may be acceptable to have a smaller imaging FOV. Compared with typical positron-emission mammography systems that use 2 planar PET detectors against the chest wall to image a breast, a breast imaging system using VP-PET geometry may allow detector 2 to be positioned farther away from the breast so that its trajectory clears the shoulder and allows the coincidence line of response to better sample tissues near the chest wall. However, this advantage may be offset by the reduced system sensitivity due to reduced solid angle coverage by detector 2 . The value of 
VP-PET in such applications remains to be seen and will require further investigation.

Finally, the VP-PET geometry has inspired us to design high-resolution insert devices to locally enhance the image resolutions of existing PET scanners, an idea similar to the use of surface coils for MRI. We have studied the potential improvement in image resolution using a full-ring insert device in a clinical whole-body PET scanner through Monte Carlo simulations (28). We are currently developing 2 PET insert systems for human and small-animal imaging applications. The potential of such devices will be investigated when the systems are completed.

\section{CONCLUSION}

We have developed and characterized a novel geometry for PET. PET systems based on this virtual-pinhole geometry exhibit certain characteristics similar to those found in a pinhole SPECT system. The potential of VP-PET geometry in creating new PET system design and new PET applications need to be further explored.

\section{ACKNOWLEDGMENTS}

The authors thank Richard Laforest, Ananya Ruangma, Martin Janecek, and Stefan Siegel for inspiring discussion; Aaron McAllister and Bill Margenau for technical support; and Dave Politte and Daniel Keesing for sharing code. This work was supported in part by the National Cancer Institute of the National Institutes of Health (grants R33-CA110011, R24-CA83060, and P30 CA91842) and by the Susan G. Komen for the Cure (grant BCTR0601279).

\section{REFERENCES}

1. Phelps ME, Hoffman EJ, Coleman RE, et al. Tomographic images of blood pool and perfusion in brain and heart. J Nucl Med. 1976;17:603-612.

2. Di Chiro G, DeLaPaz RL, Brooks RA, et al. Glucose utilization of cerebral gliomas measured by $\left[{ }^{18}\right.$ F]fluorodeoxyglucose and PET. Neurology. 1982;32: 1323-1329.

3. Beyer T, Townsend DW, Brun T, et al. A combined PET/CT scanner for clinical oncology. J Nucl Med. 2000;41:1369-1379.

4. Marriott CJ, Cadorette JE, Lecomte R, Scasnar V, Rousseau J, van Lier JE. Highresolution PET imaging and quantitation of pharmaceutical biodistributions in a small animal using avalanche photodiode detectors. J Nucl Med. 1994;35:13901396.

5. Cherry SR, Shao Y, Silverman RW, et al. MicroPET: a high resolution PET scanner for imaging small animals. IEEE Trans Nucl Sci. 1997;44:1161-1166.
6. Phelps ME. Inaugural article: positron emission tomography provides molecular imaging of biological processes. Proc Natl Acad Sci USA. 2000;97:9226-9233.

7. Moses WW, Derenzo SE. Empirical observation of resolution degradation in positron emission tomographs utilizing block detectors [abstract]. J Nucl Med. 1993;34(suppl):101P.

8. Levin CS, Hoffman EJ. Calculation of positron range and its effect on the fundamental limit of positron emission tomography system spatial resolution. Phys Med Biol. 1999;44:781-799.

9. Stickel JR, Cherry SR. High resolution PET detector design: modeling components of intrinsic spatial resolution. Phys Med Biol. 2005;50:179-195.

10. Tai Y-C, Laforest R. Instrumentation aspects of animal PET. Annu Rev Biomed Eng. 2005;7:255-285.

11. Derenzo SE, Budinger TF, Juesman RH, Cahoon JL, Vuletich T. Imaging properties of a positron tomograph with 280 BGO crystals. IEEE Trans Nucl Sci. 1981;28:81-89.

12. Chatziioannou A, Silverman RW, Meadors K, Farquhar TH, Cherry SR. Techniques to improve the spatial sampling of MicroPET: a high resolution animal PET tomograph. IEEE Trans Nucl Sci. 2000;47:422-427.

13. Rouze NC, Hutchins GD. Design and characterization of IndyPET-II: a highresolution, high-sensitivity dedicated research scanner. IEEE Trans Nucl Sci. 2003;50:1491-1497.

14. Palmer J, Wollmer P. Pinhole emission computed tomography: method and experimental evaluation. Phys Med Biol. 1990;35:339-350.

15. Cherry SR, Sorenson JA, Phelps ME. Physics in Nuclear Medicine. 3rd ed. Philadelphia, PA: Saunders; 2003.

16. Beekman FJ, van der Have F, Vastenhouw B, et al. U-SPECT-I: a novel system for submillimeter-resolution tomography with radiolabeled molecules in mice. J Nucl Med. 2005;46:1194-1200.

17. Prince JL, Jonathan LM. Medical Imaging Signals and Systems. Upper Saddle River, NJ: Pearson Prentice Hall; 2006.

18. DeBenedetti S, Cowan CE, Konneker WR, Primakoff H. On the angular distribution of two-photon annihilation radiation. Phys Rev. 1950;77:205-212.

19. Hoffman EJ, Huang S-C, Plummer D, Phelps ME. Quantitation in positron emission computed tomography. VI. Effect of nonuniform resolution. J Comput Assist Tomogr. 1982;6:987-999.

20. Derenzo SE, Moses WW, Huesman RH, Budinger TF. Critical instrumentation issues for $<2 \mathrm{~mm}$ resolution high sensitivity brain PET. In: Uemura K, Lassen NA, Jones T, Kanno I, eds. Quantification of Brain Function. Amsterdam, The Netherlands: Elsevier; 1993:25-37.

21. Siegel S, Silverman RW, Yiping S, Cherry SR. Simple charge division readouts for imaging scintillator arrays using a multi-channel PMT. IEEE Trans Nucl Sci. 1996;43:1634-1641.

22. Tai Y-C, Chatziioannou A, Siegel S, et al. Performance evaluation of the microPET P4: a PET system dedicated to animal imaging. Phys Med Biol. 2001;46:1845-1862.

23. Tomic N, Thompson CJ, Casey ME. Investigation of the "block effect" on spatial resolution in PET detectors. IEEE Trans Nucl Sci. 2005;52:599-605.

24. Tai Y-C, Ruangma A, Rowland D, et al. Performance evaluation of the microPET-Focus: a third generation microPET scanner dedicated to animal imaging. J Nucl Med. 2005;46:455-463.

25. Besson G. CT fan-beam parametrizations leading to shift-invariant filtering. Inverse Probl. 1996;12:815-833.

26. Pal D, O'Sullivan JA, Wu H, Janecek M, Tai Y-C. 2D linear and iterative reconstruction algorithms for a PET-insert scanner. Phys Med Biol. 2007;52: 4293-4310.

27. Tai Y-C, Wu HY, Janecek M. Initial study of an asymmetric PET system dedicated to breast cancer imaging. IEEE Trans Nucl Sci. 2006;53:121-126.

28. Janecek M, Wu H, Tai YC. A simulation study for the design of a prototype insert for whole-body PET scanners. IEEE Trans Nucl Sci. 2006;53:1143-1149. 